\title{
Neutrino masses and the dark energy equation of state - relaxing the cosmological neutrino mass bound
}

\author{
Steen Hannestad* \\ Department of Physics and Astronomy, University of Aarhus, Ny Munkegade, DK-8000 Aarhus C, Denmark
}

\begin{abstract}
At present cosmology provides the nominally strongest constraint on the masses of standard model neutrinos. However, this constraint is extremely dependent on the nature of the dark energy component of the Universe. When the dark energy equation of state parameter is taken as a free (but constant) parameter, the neutrino mass bound is $\sum m_{\nu} \leq 1.48 \mathrm{eV}$ (95\% C.L.), compared with $\sum m_{\nu} \leq 0.65 \mathrm{eV}(95 \%$ C.L. $)$ in the standard model where the dark energy is in the form of a cosmological constant. This has important consequences for future experiments aimed at the direct measurement of neutrino masses. We also discuss prospects for future cosmological measurements of neutrino masses.
\end{abstract}

In the past few years a new standard model of cosmology has been established in which most of the energy density of the Universe is made up of a component with negative pressure, generically referred to as dark energy. The simplest form of dark energy is the cosmological constant, $\Lambda$, which obeys $P_{\Lambda}=-\rho_{\Lambda}$. This model provides an amazingly good fit to all observational data with relatively few free parameters and has allowed for stringent constraints on the basic cosmological parameters.

The precision of the data is now at a level where observations of the cosmic microwave background (CMB), the large scale structure (LSS) of galaxies, and type Ia supernovae can be used to probe important aspects of particle physics such as neutrino properties. Conversely, cosmology is now also at a level where unknowns from the particle physics side can significantly bias estimates of cosmological parameters.

The combination of all currently available data from neutrino oscillation experiments suggests two important mass differences in the neutrino mass hierarchy. The solar mass difference of $\Delta m_{12}^{2} \simeq 7 \times 10^{-5} \mathrm{eV}^{2}$ and the atmospheric mass difference $\Delta m_{23}^{2} \simeq 2.6 \times 10^{-3} \mathrm{eV}^{2}[1]$. In the simplest case where neutrino masses are hierarchical these results suggest that $m_{1} \sim 0, m_{2} \sim \Delta m_{\text {solar }}$, and $m_{3} \sim \Delta m_{\text {atmospheric. }}$. If the hierarchy is inverted one instead finds $m_{3} \sim 0, m_{2} \sim \Delta m_{\text {atmospheric }}$, and $m_{1} \sim$ $\Delta m_{\text {atmospheric }}$. However, it is also possible that neutrino masses are degenerate, $m_{1} \sim m_{2} \sim m_{3} \gg \Delta m_{\text {atmospheric }}$. Since oscillation probabilities depend only on squared mass differences, $\Delta m^{2}$, such experiments have no sensitivity to the absolute value of neutrino masses, and if the masses are degenerate oscillation experiments are not useful for determining the absolute mass scale.

Instead, it is better to rely on kinematical probes of the neutrino mass. Using observations of the cosmic microwave background and the large scale structure of galaxies it has been possible to constrain masses of standard model neutrinos. The bound can be derived because massive neutrinos contribute to the cosmological matter density, but they become non-relativistic so late that any perturbation in neutrinos up to scales around the causal horizon at matter-radiation equality is erased, i.e. the kinematics of the neutrino mass influences the growth of structure in the Universe. Quantitatively, neutrino free streaming leads to a suppression of fluctuations on small scales relative to large by roughly $\Delta P / P \sim-8 \Omega_{\nu} / \Omega_{m}$ [2]. The density in neutrinos is related to the number of massive neutrinos and the neutrino mass by

$$
\Omega_{\nu} h^{2}=\frac{\sum m_{\nu}}{93.2 \mathrm{eV}}=\frac{N_{\nu} m_{\nu}}{93.2 \mathrm{eV}}
$$

if all neutrinos are assumed to have the same mass. $h$ is the Hubble parameter in units of $100 \mathrm{~km} \mathrm{~s}^{-1} \mathrm{Mpc}^{-1}$. Such an effect would be clearly visible in LSS measurements, provided that the neutrino mass is sufficiently large, and a likelihood analysis based on the standard $\Lambda \mathrm{CDM}$ model with neutrino mass as an added parameter in general provides a bound for the sum of neutrino masses of roughly $\sum m_{\nu} \leqslant 0.5-1 \mathrm{eV}$, depending on exactly which data is used [3, 4].

This should be compared to the present laboratory bound from ${ }^{3} \mathrm{H}$ beta decay found in the Mainz experiment, $m_{\nu_{e}}=\left(\sum_{i}\left|U_{e i}\right|^{2} m_{i}^{2}\right)^{1 / 2} \leq 2.3 \mathrm{eV}[\underline{5}$ ]. It should also be contrasted to the claimed signal for neutrinoless double beta decay in the Heidelberg-Moscow experiment [6], which would indicate a value of 0.1-0.9 $\mathrm{eV}$ for the relevant combination of mass eigenstates, $m_{e e}=\left|\sum_{j} U_{e j}^{2} m_{\nu_{j}}\right|$. Some papers claim that the cosmological neutrino mass bound is already incompatible with this measurement.

However, as with almost all likelihood analyses of parameters beyond those in the simplest $\Lambda \mathrm{CDM}$ model, it is based on a relatively limited parameter space. Using a much more complicated model with a non-power law primordial power spectrum it is possible to accomodate large neutrino masses, provided that Type Ia supernova data is discarded [7]. Here, instead, we provide a very simple (from a cosmological point of view) extension of the standard $\Lambda \mathrm{CDM}$ model in which the dark energy component, $X$, is represented by a fluid with a more general equation of state, $P_{X}=w \rho_{X}$. For simplicity we take $w$ to be a constant. Such models have been studied 
extensively in the literature [8] and many of them are motivated by scalar field models, or models with modified gravity on large scales. Most dark energy models have $w \geq-1$, but it is possible to construct models with $w<-1$ from non-standard kinetic terms in string theory or from models with modified gravity 9 ].

In fact, it is by now customary in almost all papers on cosmological parameter fitting to allow $w<-1$. Here, we also allow $w$ to take values below -1 in the so-called phantom energy regime, and perform a standard likelihood analysis for this model. As will be seen below, this relatively simple extension of the $\Lambda \mathrm{CDM}$ model vastly decreases the precision with which present observations can constrain neutrino masses.

Likelihood analysis - We have performed a likelihood analysis using the most recent observational data from cosmology. We use the CMB measurements by the Wilkinson Microwave Anisotropy Probe (WMAP) satellite [3], the galaxy power spectrum provided by the Sloan Digital Sky Survey (SDSS) collaboration 10], and the type Ia supernova data from Riess et al 11].

As parameters in the likelihood analysis we use a standard flat, dark energy dominated model with the following free parameters: $\Omega_{\mathrm{CDM}}$, the CDM density, $\Omega_{b}$, the baryon density, $H_{0}$, the Hubble parameter, $n_{s}$, the scalar spectral index of the primordial power spectrum, $\tau$, the optical depth to reionization, $Q$, the normalization of the CMB spectrum, $b$, the bias parameter, and $w=P_{X} / \rho_{X}$, the equation of state of the dark energy. Finally we also use the contribution of neutrinos, $\Omega_{\nu} h^{2}=\frac{\sum m_{\nu}}{93.2 \mathrm{eV}}$. From here on we assume three neutrinos with degenerate masses so that $\Omega_{\nu} h^{2}=\frac{3 m_{\nu}}{93.2 \mathrm{eV}}$. From the flatness criterion this gives the dark energy density as $\Omega_{X}=1-\Omega_{\mathrm{CDM}}-\Omega_{b}-\Omega_{\nu}$. As additional data we use the data from the Hubble Space Telescope (HST) Key Project on $H_{0}$ which give $H_{0}=72 \pm 8 \mathrm{~km} \mathrm{~s}^{-1} \mathrm{Mpc}^{-1}$ [12]. We allow the bias parameter $b$ to vary freely. While it is possible to obtain stronger bounds on neutrino masses by adding information on the bias parameter, this information is most likely dominated by systematics and the errors correspondingly difficult to quantify.

In Fig. 1 we show results for $\Delta \chi^{2}=\chi^{2}-\chi_{0}^{2}$ as a function of neutrino mass, marginalized over the other relevant parameters ( 7 if $w$ is kept constant, 8 otherwise). The marginalization was performed using a simulated annealing algorithm 13. The normalization constant $\chi_{0}^{2}$ is taken to be that of the best fit model in each case. For a free $w$ the best fit model has $\chi_{0}^{2}=1625.5$ for 1513 degrees of freedom $\left(\chi^{2} /\right.$ d.o.f $\left.=1.074\right)$, and for fixed $w=-1$ the best fit has $\chi_{0}^{2}=1626.9$ for 1514 degrees of freedom $\left(\chi^{2} /\right.$ d.o.f $\left.=1.075\right)$. Note that for 1-dimensional parameter constraints we take the $95 \%$ C.L. to be $\Delta \chi^{2}=4$. For 2-dimensional parameter estimates we take the $68 \%$ C.L. at $\Delta \chi^{2}=2.31$ and the $95 \%$ C.L. at $\Delta \chi^{2}=6.17$.

From the figure, and from table I (the bottom line), it

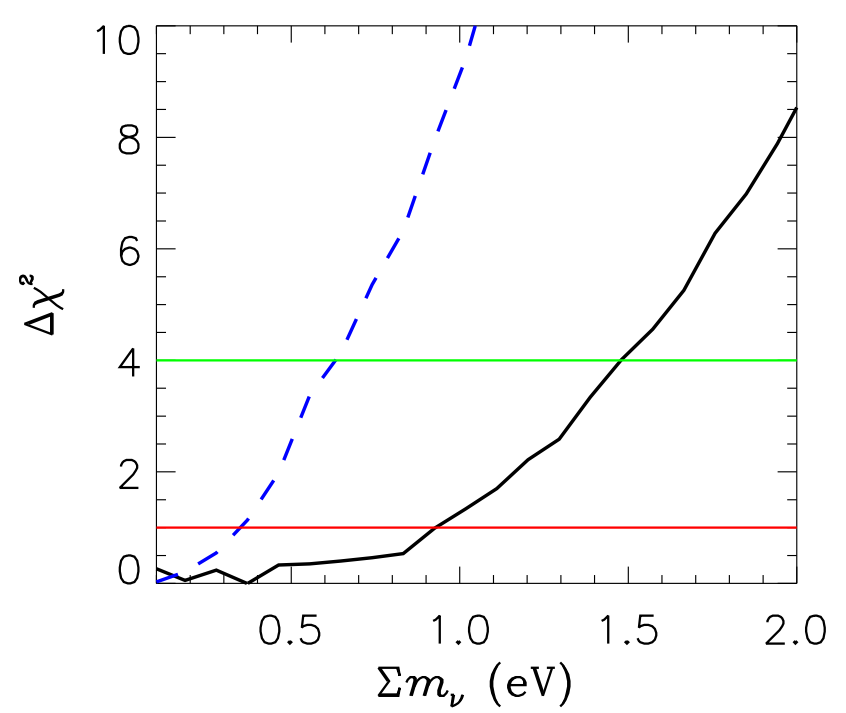

FIG. 1: $\Delta \chi^{2}$ as a function of neutrino mass for various different data sets and parameter assumptions. The dashed line is for a fixed $w=-1$, using WMAP, SDSS, HST, and SNI-a data. The full line is for a free $w$ with the same data. The horizontal lines show $\Delta \chi^{2}=1$ and 4 , corresponding to $68 \%$ and $95 \%$ C.L. respectively.

TABLE I: The 95\% C.L. upper bound on the sum of neutrino masses from present cosmological observations.

\begin{tabular}{lc}
\hline \hline Data used & $\sum m_{\nu}$ \\
\hline WMAP+SDSS+HST+SNI-a & $1.48 \mathrm{eV}$ \\
WMAP+SDSS+HST+SNI-a (fixed $w)$ & $0.65 \mathrm{eV}$ \\
\hline \hline
\end{tabular}

is clear that with $w=-1$ we retrieve the known result, $\sum m_{\nu} \leq 0.65 \mathrm{eV}$ (95\% C.L.), published in the literature for the same data 14. However, as soon as $w$ is allowed to vary the mass bound degrades tremendously, and in fact allows very high neutrino masses. For the data used here the bound is $\sum m_{\nu} \leq 1.48 \mathrm{eV}$ (95\% C.L.). Note that if neutrino masses are close to saturating this bound they will be easily detectable by the KATRIN experiment, which has a projected sensitivity of $0.2 \mathrm{eV}$ for the effective electron neutrino mass [15], corresponding roughly to $\sum m_{\nu} \simeq 0.6 \mathrm{eV}$. It is also completely compatible with the claimed detection of a non-zero neutrino mass by the Heidelberg-Moscow experiment, with a best fit around $m_{e e} \simeq 0.1-0.9 \mathrm{eV}[\underline{6}$.

In Fig. 2 we show the analysis in a grid for both $\sum m_{\nu}$ and $w$. From this it can be seen that there is an almost perfect degeneracy between these two parameters, an increasing $\sum m_{\nu}$ can be compensated by decreasing $w$. While for low neutrino masses a cosmological constant $(w=-1)$ is allowed, for high neutrino masses only dark energy models in the phantom regime $(w<-1)$ are allowed. 


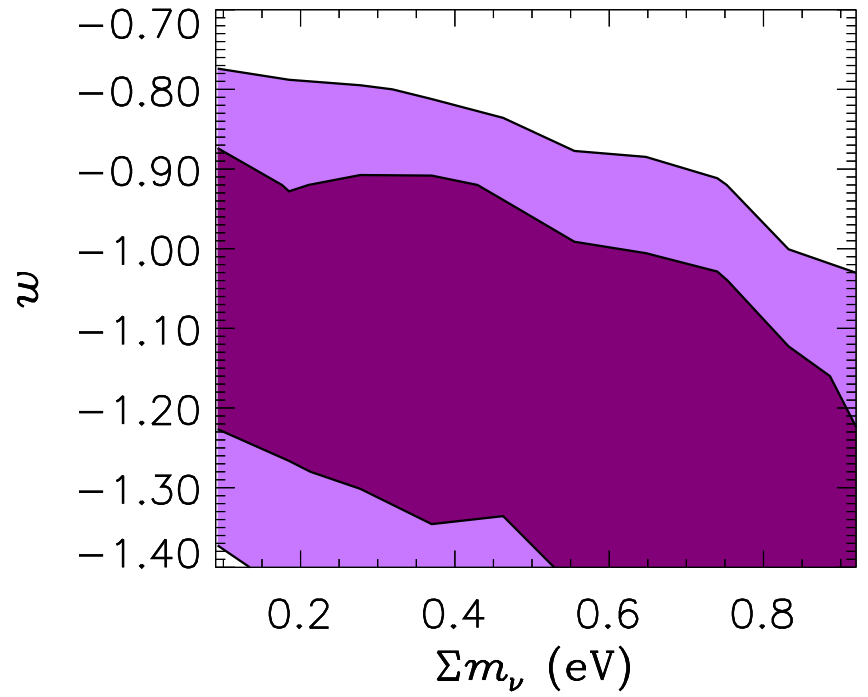

FIG. 2: $68 \%$ and $95 \%$ allowed contours as a function of neutrino mass and dark energy equation of state using WMAP, SDSS, HST, and SNI-a data.

The reason for the degeneracy is that when $\Omega_{\nu}$ is increased, $\Omega_{m}$ must be increased correspondingly in order to produce the same power spectrum. However, when $w=-1$ an increasing $\Omega_{m}$ quickly becomes incompatible with the supernova data. This can be remedied by simultaneously decreasing $w$ because of the well-known $\Omega_{m}, w$ degeneracy in the supernova data. This effect can be seen in Fig. 3: If $w$ is allowed to vary freely, $\Omega_{m}$ can take very high values without being inconsistent with the supernova data, because in this case the $w=-1$ upper bound on $\Omega_{m}$ does not apply. As soon as the model with fixed $w=-1$ approaches $\sigma m_{\nu} \sim 0.7 \mathrm{eV}$ and the best fit $\Omega_{m}$ crosses the Riess et al. bound 11], the model becomes strongly disfavoured.

This model gives probably the simplest example of how to relax the very stringent cosmological neutrino mass bound. Other means have been discussed in the literature, such as broken scale invariance [] or mass-varying neutrinos [16], but this is by far the simplest scenario yet discussed.

Future constraints - Since present data clearly do not give very stringent constraints on $\sum m_{\nu}$ in the presence of phantom energy, it is worthwhile discussing whether future data will be able to break the degeneracy. For that purpose we have performed a Fisher matrix analysis based on a reference model with the same free parameters as in our fit to present data $\left(\Omega_{\mathrm{CDM}} h^{2}, w_{X}, \Omega_{b} h^{2}, \Omega_{X}, n_{s}, \tau, Q, b, \sum m_{\nu}\right)$. The parameters in the reference model are $(0.1225,-1,0.0245,0.7,1,0.05,1,1,0.05 \mathrm{eV}) . \quad$ Note that we again assume three massive neutrinos with completely degenerate masses. While this approximation

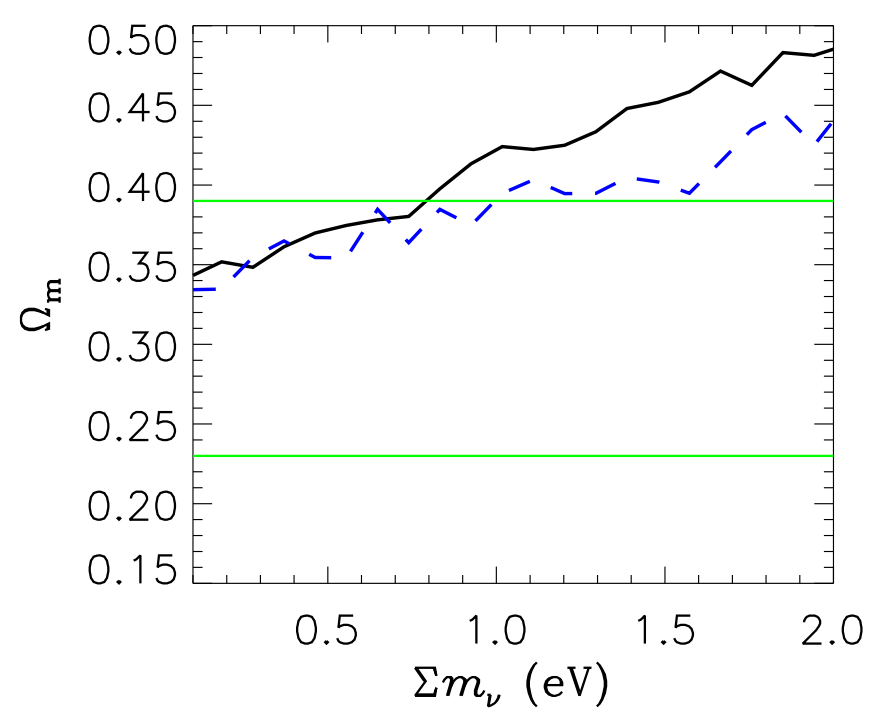

FIG. 3: The value of $\Omega_{m}$ for the best fit models, as a function of $\sum m_{\nu}$. The curve labels are the same as in Fig. 1. The horizontal (red) lines are the $2 \sigma$ bounds from the present Riess et al. supernova data [1] for the case of $w=-1$.

is valid as long as $\sum m_{\nu} \gtrsim 0.15 \mathrm{eV}$, it breaks down for smaller masses. This introduces a relatively small numerical error, and does not qualitatively change the results (see also 21] for a discussion of this point).

The Fisher matrix analysis is based on the second derivatives of the likelihood function around the reference model and allows for an estimate of the $1 \sigma$ error bars on measurements of all the relevant parameters in a given experiment, assuming that errors are Gaussian. For the future CMB data we assume an experiment which measures both temperature and $E$-type polarization. It is assumed to be limited by cosmic variance up to $l=2500$ for temperature and $l=1500$ for polarization, i.e. an experiment slightly better than the upcoming Planck satellite 17. (hereafter we call this hypothetical experiment Planck + ). We neglect $B$-type polarization even though it will most likely be measured by Planck. Adding information on $B$-type polarization would not significantly alter the results. For a future LSS survey we assume an effective survey volume of $V=\frac{4}{3} \pi \lambda^{3}$ with $\lambda=1000 h^{-1} \mathrm{Mpc}$ (see [18] for a discussion). This should be compared to the SDSS-BRG 19] survey which has $\lambda \simeq 620 h^{-1}$ Mpc. We also assume that the linear power spectrum can be inferred with only sampling error up to a wavenumber of $k=0.15 h / \mathrm{Mpc}$. In table II we show the $1 \sigma$ error bars which can be expected on $w$ and $\sum m_{\nu}$ for such a combination of data.

From the table it is clear that if either $w$ or $\sum m_{\nu}$ can be assumed to be fixed then a very stringent constraint can be obtained on the other parameter. However, as soon as both parameters are allowed to very freely the 
TABLE II: Estimated $1 \sigma$ errors on $w$ and $\sum m_{\nu}$ for the Planck + SDSS data set. The errors have been calculated from a Fisher matrix analysis, as described in the text.

\begin{tabular}{lcc}
\hline \hline & fixed $w$ or $m_{\nu}$ & free $w$ and $m_{\nu}$ \\
\hline$\sigma\left(\sum m_{\nu}\right)$ & $0.106 \mathrm{eV}(w$ fixed $)$ & $0.288 \mathrm{eV}$ \\
$\sigma(w)$ & $0.026\left(m_{\nu}\right.$ fixed $)$ & 0.069 \\
\hline \hline
\end{tabular}

error bars blow up by a factor of almost 3 . The estimate that $\sigma\left(\sum m_{\nu}\right) \sim 0.06-0.07 \mathrm{eV}[20]$, is clearly too optimistic unless additional information on $w$ is provided (see also [21] for further discussion). Such information could for instance come from large scale weak lensing surveys [22], or from measurements by the SNAP supernova survey satellite [23].

Conversely, in order to provide a stringent constraint on $w$ it is necessary to obtain prior knowledge about the neutrino masses. This could come from experiments such as KATRIN [15] which is designed to probe the effective electron neutrino mass to a precision of about $0.2 \mathrm{eV}$, or from neutrinoless double beta decay experiments which in theory are sensitive to the sub- $0.1 \mathrm{eV}$ range.

Conclusion - We have studied the cosmological neutrino mass bound in cosmological models where the dark energy equation of state is allowed to take on an arbitrary, but constant value. We find that this relaxes the present cosmological bound on neutrino masses by more than a factor of two, to $\sum m_{\nu} \leq 1.48 \mathrm{eV}$ at $95 \%$ C.L. Furthermore, even with the much more precise CMB and LSS data available in the future, the degeneracy persists unless additional data from weak lensing or similar probes can be used to break it.

The example provided in this paper clearly illustrates that while cosmological bounds on particle physics parameters are very impressive, they are also model dependent. A cosmological neutrino mass bound cannot stand completely alone, it should be complemented by direct laboratory measurements in the $0.1 \mathrm{eV}$ sensitivity range.

Acknowledgement - Use of the publicly available CMBFAST package is acknowledged [24].

* Electronic address: sth@phys.au.dk

[1] P. Aliani, V. Antonelli, M. Picariello and E. TorrenteLujan, hep-ph/0309156 P. C. de Holanda and A. Y. Smirnov, Astropart. Phys. 21, 287 (2004); M. Maltoni, T. Schwetz, M. A. Tortola and J. W. F. Valle, Phys. Rev. D 68, 113010 (2003).

[2] W. Hu, D. J. Eisenstein and M. Tegmark, Phys. Rev. Lett. 80 (1998) 5255.

[3] C. L. Bennett et al., Astrophys. J. Suppl. 148 (2003) 1; D. N. Spergel et al., Astrophys. J. Suppl. 148 (2003) 175

[4] S. Hannestad, JCAP 0305 (2003) 004; O. Elgaroy and O. Lahav, JCAP 0304 (2003) 004; V. Barger, D. Mar- fatia and A. Tregre, hep-ph/0312065 S. Hannestad and G. Raffelt, JCAP 0404, 008 (2004); P. Crotty, J. Lesgourgues and S. Pastor, Phys. Rev. D 69, 123007 (2004); S. Hannestad, hep-ph/0404239 U. Seljak et al., astro-ph/0407372 G. L. Fogli, E. Lisi, A. Marrone, A. Melchiorri, A. Palazzo, P. Serra and J. Silk, Phys. Rev. D 70, 113003 (2004); S. Hannestad, hep-ph/0409108 M. Tegmark, hep-ph/0503257

[5] C. Kraus et al. European Physical Journal C (2003), proceedings of the EPS 2003 - High Energy Physics (HEP) conference.

[6] H. V. Klapdor-Kleingrothaus, I. V. Krivosheina, A. Dietz and O. Chkvorets, Phys. Lett. B 586, 198 (2004).

[7] A. Blanchard, M. Douspis, M. Rowan-Robinson and S. Sarkar, Astron. Astrophys. 412, 35 (2003)

[8] R. Bean and A. Melchiorri, Phys. Rev. D 65, 041302 (2002); S. Hannestad and E. Mortsell, Phys. Rev. D 66, 063508 (2002); A. Melchiorri, L. Mersini, C. J. Odman and M. Trodden, Phys. Rev. D 68, 043509 (2003).

[9] R. R. Caldwell, Phys. Lett. B 545, 23 (2002); S. M. Carroll, M. Hoffman and M. Trodden, Phys. Rev. D 68, 023509 (2003); G. W. Gibbons, hep-th/0302199 R. R. Caldwell, M. Kamionkowski and N. N. Weinberg, Phys. Rev. Lett. 91, 071301 (2003); S. Nojiri and S. D. Odintsov, Phys. Lett. B 562, 147 (2003); M. G. Brown, K. Freese and W. H. Kinney, arXiv:astro-ph/0405353 V. K. Onemli and R. P. Woodard, Phys. Rev. D 70, 107301 (2004); C. Deffayet, G. R. Dvali and G. Gabadadze, Phys. Rev. D 65, 044023 (2002); G. Dvali and M. S. Turner, arXiv:astro-ph/0301510 L. Knox, Y. S. Song and J. A. Tyson, arXiv:astro-ph/0503644

[10] M. Tegmark et al., Phys. Rev. D 69, 103501 (2004); M. Tegmark et al., Astrophys. J. 606, 702 (2004)

[11] Riess, A. G., et al. 2004, ApJ, 607, 665

[12] W. L. Freedman et al., Astrophys. J. Lett. 553, 47 (2001).

[13] S. Hannestad, Phys. Rev. D 61 (2000) 023002.

[14] S. Hannestad, hep-ph/0409108

[15] See the KATRIN 2005 design report at http://www-ik.fzk.de/katrin/

[16] R. Fardon, A. E. Nelson and N. Weiner, JCAP 0410, 005 (2004); R. D. Peccei, Phys. Rev. D 71, 023527 (2005) X. J. Bi, B. Feng, H. Li and X. m. Zhang, arXiv:hep-ph/0412002

[17] http://astro.estec.esa.nl/Planck/

[18] M. Tegmark, Phys. Rev. Lett. 79, 3806 (1997).

[19] http://www.sdss.org

[20] S. Hannestad, Phys. Rev. D 67, 085017 (2003).

[21] J. Lesgourgues, S. Pastor and L. Perotto, Phys. Rev. D 70, 045016 (2004);

[22] S. Wang, Z. Haiman, W. Hu, J. Khoury and M. May, astro-ph/0505390 K. N. Abazajian and S. Dodelson, Phys. Rev. Lett. 91, 041301 (2003); M. Kaplinghat, L. Knox and Y. S. Song, Phys. Rev. Lett. 91, 241301 (2003)

[23] http://snap.lbl.gov/

[24] U. Seljak and M. Zaldarriaga, Astrophys. J. 469 (1996) 437 See also the CMBFAST website at http://www.cmbfast.org 